\title{
Motor aging results from cerebellar neuron death
}

\author{
Matthieu P. Boisgontier \\ Trends in Neurosciences (2015) \\ DOI: 10.1016/j.tins.2015.01.003
}

Movement Control and Neuroplasticity Research Group, Department of Kinesiology, Biomedical Sciences Group, KU Leuven, Leuven, Belgium

Correspondence: matthieu.boisgontier@faber.kuleuven.be

\begin{abstract}
As we age movements become slower, inconsistent and require more attention. These hallmarks of aging suggest a switch from predictive to reactive motor control. Here, I examine evidence supporting the hypothesis that motor aging is primarily determined by the early death of neurons in the cerebellum, a critical structure for predictive motor control.
\end{abstract}

\section{Keywords}

Aging; Cerebellum; Motor control 


\section{Motor aging results from cerebellar neuron death}

In our rapidly aging society, functional independence is a critical challenge for the health and well-being of individuals and has considerable socioeconomic consequences. In this regard, the ability to control movement is crucial. Therefore, to succeed in promoting longer functional independence we need to understand the normal aging process of movement control. Such motor aging is obviously multifactorial and complex. However, specific structures may have a prevailing role. If this is the case, targeting these structures may allow more efficient interventions. Here, I examine evidence supporting the hypothesis that motor aging is primarily determined by an early death of cerebellar neurons.

Populations of neurons in the cerebrum and cerebellum are not equally affected by normal aging. Indeed, the number of neurons is stable in numerous cerebral areas including the primary motor cortex, primary visual cortex, prefrontal cortex, hippocampus, and entorhinal cortex [1]. Conversely, the cerebellum displays a significant loss of neurons. The anterior lobe is especially affected with a $40 \%$ loss of both Purkinje and granule cells [2]. This difference in neuron loss between the cerebrum and cerebellum may be explained by the vulnerability of cerebellar neurons. Specifically, Purkinje cells show a high and premature susceptibility to mitochondrial and proteostasis defects [3], and cerebellar granule cells are particularly vulnerable to oxidative stress [4].

What makes the cerebellum critical for motor control is its ability to estimate both extrinsic and intrinsic forces acting on the body. Based on these estimates, movement is controlled before availability of sensory reafferences. While such predictive motor control supported by 
Boisgontier MP (2015) Motor aging results from cerebellar neuron death. Trends in Neurosciences. [In press] DOI: 10.1016/j.tins.2015.01.003

the cerebellum was suggested a long time ago [5], neurophysiological evidence started to appear only recently. Specifically, Purkinje cells output an estimate of physical principles acting on the body such as gravity [6]. Granule cells potentially integrate a copy of a motor command and sensory inputs from the spinocerebellar tract to estimate the sensory consequence of the motor command [7]. This predictive control has consistently shown to be impaired after cerebellar damage [8]. It follows that the death of cerebellar neurons observed in normal aging impairs the predictive control of movement.

Here, I propose that in older adults early cell loss prevents the cerebellum from outputting accurate estimates of intrinsic and extrinsic forces acting on the body, thereby increasing the computational load required to perform a motor task at the same level as young adults. As age-related neuron death appears to prominently affect the cerebellum, the resulting impairment of predictive motor control is likely to have a major role in motor aging. The high percentage of neuron loss in the anterior lobe, a cerebellar region critical for motor control, further supports this hypothesis.

At the brain level, this additional load is evidenced by age-related overactivation, which has been consistently observed in regions involved in skilled movement such as the prefrontal cortex, presupplementary motor area, premotor cortex, parietal cortex, and supplementary motor area [9]. Furthermore, cerebellar overactivation [9] suggests that the remaining neurons operate less efficiently. At the behavioral level, the additional computational load is evidenced by a declining ability to perform a motor task concurrently with another task [10].

If cerebellar neuron death primarily determines motor aging as hypothesized, the behavior of older adults should reflect impaired predictive motor control. In fact, slowness and 
inconsistency, the hallmarks of movement in older adults perfectly match the behavior expected in the context of impaired predictive control. Indeed, the direct consequence of impaired predictive control would be an increased reliance on reactive control, which is based on time-delayed sensory feedback. Due to such delays, movement corrections are always late with regard to the portion of movement they intend to correct. Accordingly, individuals with impaired predictive motor control are expected to reach their goal through a series of corrective movements. Such behavior has consistently been found in older adults [11]. Furthermore, direct consequences of time-delayed feedback and corrective movements are slowness and inconsistency.

In sum, there is consistent evidence supporting the hypothesis that motor aging is primarily determined by an early death of cerebellar neurons. To delay motor aging and promote longer functional independence, future work investigating the cerebellum and means to compensate for this cellular death is warranted. In this regard, a recent study demonstrating enhanced motor adaptation in older adults following transcranial direct current stimulation over the cerebellum is encouraging [12]. Finally, the critical role of the cerebellum in motor aging does not exclude the involvement of other brain structures.

\section{Acknowledgment}

Matthieu P. Boisgontier is supported by a research grant $(1504015 \mathrm{~N})$ and a post-doctoral fellowship of the Research Foundation - Flanders (FWO).

\section{References}

[1] Peters, A. and Kemper, T. (2012) A review of the structural alterations in the cerebral hemispheres of the aging rhesus monkey. Neurobiol. Aging 33, 2357-2372. 
Boisgontier MP (2015) Motor aging results from cerebellar neuron death. Trends in Neurosciences. [In press] DOI: 10.1016/j.tins.2015.01.003

[2] Andersen, B.B., et al. (2003) Aging of the human cerebellum: A stereological study. J. Comp. Neurol. 466, 356-365.

[3] Hekman, K.E. and Gomez, C.M. (2015) The autosomal dominant spinocerebellar ataxias: Emerging mechanistic themes suggest pervasive Purkinje cell vulnerability. J. Neurol. Neurosurg. Psychiatry (in press)

[4] Wang, X. and Michaelis, E.K. (2010) Selective neuronal vulnerability to oxidative stress in the brain. Front. Aging Neurosci. 2:12

[5] Wolpert, D.M. et al. (1998) Internal models in the cerebellum. Trends Cogn. Sci. 2, 338347

[6] Laurens J. et al. (2013) Computation of linear acceleration through an internal model in the macaque cerebellum. Nat. Neurosci. 16, 1701-1708

[7] Huang, C.C. et al. (2013) Convergence of pontine and proprioceptive streams onto multimodal cerebellar granule cells. Elife 2: e00400

[8] Bastian, A.J. (2006) Learning to predict the future: The cerebellum adapts feedforward movement control. Curr. Opin. Neurobiol. 16, 645-649

[9] Heuninckx, S. et al. (2005) Neural basis of aging: The penetration of cognition into action control. J. Neurosci. 25, 6787-6796

[10] Verhaeghen, P. et al. (2003) Aging and dual-task performance: A meta-analysis. Psychol. Aging 18, 443-460

[11] Boisgontier, M.P. and Nougier, V. (2013) Ageing of internal models: From a continuous to an intermittent proprioceptive control of movement. Age 35, 1339-1355

[12] Hardwick, R.M. and Celnik, P.A. (2014) Cerebellar direct current stimulation enhances motor learning in older adults. Neurobiol. Aging 35, 2217-2221 